\title{
The Rep-PCR typing of TEM type ESBL producing clinical isolates of klebsiella pneumonia
}

\begin{abstract}
Objective: The aims of this study were to detect and fingerprint TEM-producing clinical isolates of $\mathrm{K}$ pneumonia.

Materials and methods: In this cross-sectional study from July 2013-May 2015, a total of $200 \mathrm{~K}$. pneumonia isolates were collected from various origins (all from hospital acquired infections) in Tehran, Iran. The antibiotic susceptibility and MIC of isolates were performed according to the CLSI guidelines. The combined disk method was employed to determine ESBL producer isolates. PCR was done for detection of the gene encoding TEM type enzyme. The rep-PCR technique was applied for the fingerprinting of $\mathrm{K}$. pneumonia isolates.
\end{abstract}

Results: The age range of patients was $1-69$ years (mean $=41.33$, including $93(46.5 \%)$ males and $107(53.5 \%)$ females). Ninety ( $45 \%$ ) isolates exhibited MIC $\geq 2$ to ceftazidime and cefotaxime, respectively, and $87(43.5 \%)$ demonstrated ESBL production. The highest and lowest resistance was against erythromycin and imipenem $(93.5 \%$ and $0 \%$, respectively). The prevalence of bla TEM gene among ESBL producers was $87.3 \%(\mathrm{n}=76)$. The rep-PCR typing of isolates showed diversity but a $90 \%$ similarity among TEM producing isolates, suggesting polyclonal spread of TEM type producing isolates.

Conclusion: The findings of the current study concluded the emergence and spread of $\mathrm{K}$. pneumonia isolates producing TEM type enzymes with a genetically diverse background in Tehran.

Keywords: klebsiella pneumonia, tem type esbls, genotyping
Volume 4 Issue 4 - 2017

\author{
Abdolmajid Ghasemian,' Farshad Nojoomi,' \\ Majid Eslami² \\ 'Department of Microbiology, AJA University of Medical \\ Sciences, Iran \\ ${ }^{2}$ Department of Bacteriology, Tarbiat Modares University, Iran
}

Correspondence: Majid Eslami, Department of Microbiology, AJA University of Medical Sciences, Iran,

Email bacteriology94@gmail.com

Received: August 08, 2017 | Published: August II, 2017

\section{Introduction}

Extended-spectrum Beta-lactamase (ESBL) producing $\mathrm{K}$ pneumonia has been considered among six drug-resistant microbes to which new therapies are urgently needed. ${ }^{1}$ Increasing resistance in K. pneumoniae to first-line antibiotics have made therapeutic options for urinary tract (UTIs) and other infections challenging. ${ }^{2}$ The reports of ESBLs from K. pneumonia have been sharply increased since the years 2009-2010 until today. ${ }^{3,4}$ TEM- and SHV-ESBLs, with over 100 mutations being reported, are major cause of hospitalacquired infections, particularly in the Intensive Care Unit (ICU). The blaTEM genes are parts of the Tn3 transposons elements, namely, Tn3 (blaTEM-1a), Tn1/Tn801 (blaTEM-2) and Tn2 (blaTEM-1b), which are carried by highly transmissible plasmids. ${ }^{5,6}$ Although TEM1 was considered as the most frequent gene among ESBL producers in 1980s and early 1990, today there are reports showing SHV-1 as the most prevalent ESBL gene in many parts of the world. ${ }^{7}$ It has been supposed that the naturally occurring TEM-type ESBLs are the outcome of fluctuating selective pressure due to several betalactams within a given ward or hospital rather than selection with a single agent. ${ }^{8}$ Repetitive estrogenic Palindromic elements (REP) are short sequences mostly described in gram-negative species. ${ }^{9}$ The prevalence of K. pneumonia TEM type ESBLs among hospitalized patients in Iran have been reported as following: $32 \%(\mathrm{n}=18)$ from Tehran in $2007,{ }^{10} 35.29 \%(\mathrm{n}=18)$ from Tehran in $2008,{ }^{11} 54 \%(\mathrm{n}=48)$ from Tehran in $2010,{ }^{12} 8 \%(\mathrm{n}=7)$ from Tehran in $2010,{ }^{13} 15.38 \%$ $(\mathrm{n}=4)$ from Ahvaz in 2013, 30.7\% $(\mathrm{n}=32)$ from Tehran in $2010^{14}$ and $39 \%(n=18)$ from Shiraz in 2013..$^{15}$ Several studies have shown that rep-PCR has the ability to fingerprint strains of Escherichia coli and
K. pneumonia and other gram-negative species. ${ }^{16-19}$ There has been excellent correlation between MLST and automated rep-PCR in K. pneumonia fingerprinting. ${ }^{20}$ The aims of this study were to detect and fingerprint TEM-producing K. pneumonia isolates from hospitalized patients in Tehran.

\section{Material and methods}

\section{Bacterial strains and cultures}

In this cross-sectional study from July 2013- May 2015, a total of $200 \mathrm{~K}$. pneumonia isolates were collected from hospitalized patients with age range of $1-69$ years (mean $=41.33$, including $93(46.5 \%)$ males and 107 (53.5\%) females) in Tehran, Iran during 2013-2015. The isolates were identified by biochemical tests and subsequently were stored in Trypticase soy broth containing $30 \%$ glycerol at $-20^{\circ} \mathrm{C}$.

Antibiotic susceptibility testing The antibiotic susceptibility testing was conducted with disk diffusion method (Kirby-Bauer) according to CLSI recommendation using 14 antibiotics, including FOX $(30 \mu \mathrm{g})$, CAZ $(30 \mu \mathrm{g})$, CTX $(30 \mu \mathrm{g})$, CPM $(50 \mu \mathrm{g})$, ATM $(50 \mu \mathrm{g})$, ERY $(15 \mu \mathrm{g}), \mathrm{GM}(10 \mu \mathrm{g}), \mathrm{TE}(30 \mu \mathrm{g})$, SXT $(25 \mu \mathrm{g}), \mathrm{AX}(30 \mu \mathrm{g}), \mathrm{AM}$ $(25 \mu \mathrm{g})$, IPM $(10 \mu \mathrm{g}), \mathrm{AN}(30 \mu \mathrm{g})$ and CP $(30 \mu \mathrm{g})$ (from MAST, UK), determined. E. coli ATCC 25922 was used to control antibiogram and combined disk. ${ }^{21}$

\section{Phenotypic ESBL production}

ESBL production was assessed by using combined disk method, in which cefotaxime $(30 \mu \mathrm{g})$ and ceftazidime $(30 \mu \mathrm{g})$ with and without the Clavulanic acid were used. After incubation for 24 hours in $37^{\circ} \mathrm{C}$ 
ESBL producing by increasing the size of inhibition zone diameter $\geq 5 \mathrm{~mm}$ to Cefotaxime and Ceftazidime in the combination of each of them with Clavulanic acid were an indicator of ESBL production. ${ }^{22}$

\section{Minimum inhibitory concentration (MIC)}

The agar dilution MIC method with and without Clavulanic acid inhibitor to Cefotaxime and Ceftazidime in concentrations serial 0.5 to $512 \mu \mathrm{g} / \mathrm{ml}$ was used. E. coli ATCC25922 was used to control bacteria. MIC in the presence of $4 \mu \mathrm{g} / \mathrm{ml}$ of Clavulanic acid inhibitor was used in all plates.

\section{DNA extraction}

$\mathrm{K}$. pneumonia isolates were cultured on trypticase soy agar for $24 \mathrm{~h}$ at $37^{\circ} \mathrm{C}$. DNA from each strain was extracted using the Cinagen kit according to the manufacturer's instruction. DNA yield was determined based on absorbance at $260 \mathrm{~nm}$ using a spectrophotometer and stored at $-20^{\circ} \mathrm{C}$.

\section{PCR detection of blaTEM gene}

TEM (F) 5'-ATG AGT ATT CAA CAT TTC CG-3' and TEM (R) 5'-CCA ATG CTT AAT CAG TGA GG-3' primers were used for the amplification of genes coding for TEM type $\beta$-lactamase. Amplification was performed in a final volume of $25 \mu$ l containing $0.5 \mathrm{U}$ of Taq DNA polymerase, $2.5 \mu 1$ of $10 \times$ buffers, $2.5 \mu 1 \mathrm{dNTPs}, 2 \mu 1$ primers (12.5pmol each), and $2.5 \mu \mathrm{l}$ DNA template. Reactions were run with an eppendorfs PCR system under the following conditions: $4 \mathrm{~min}$ at $94^{\circ} \mathrm{C}$, followed by 35 cycles of $1 \mathrm{~min}$ at $94^{\circ} \mathrm{C}, 1 \mathrm{~min}$ at $55^{\circ} \mathrm{C}$, and $1 \mathrm{~min}$ at $72^{\circ} \mathrm{C}$, followed by a final extension of $10 \mathrm{~min}$ at $72^{\circ} \mathrm{C}$. The PCR products were electrophoreses on $1 \%$ agarose gel (sigma), stained with ethidium bromide, and photographed under UV light.

\section{Rep-PCR typing}

DNA was subjected to PCR utilizing the primers REP 1R-I (5'- IIIICGICGICATCIGGC -3') and REP 2-I (5'ICGICTTATCIGGCCTAC $-3^{\prime}$ ) as described by Versalovic et al. ${ }^{23}$ Amplification reactions were performed in a final volume of $25 \mu 1$ containing $45 \mathrm{pmol}(1.8 \mu \mathrm{mol} . \mathrm{L}-1)$ and $36 \mathrm{pmol}(1.44 \mu \mathrm{mol} . \mathrm{L}-1)$ of each of the REP primers, $0.200 \mathrm{mmol} . \mathrm{L}-1$ of each deoxynucleoside triphosphate, 10mmol.L-1 Tris-HCL (PH8.3), 1.5mmol.L-1 $\mathrm{MgCl}_{2}$ and $0.35 \mathrm{U}$ of Taq DNA polymerase. Amplification was performed in a PCR thermal cycler, using the following temperature profile: $95^{\circ} \mathrm{C}$ for $6 \mathrm{~min}, 30$ cycles at $94^{\circ} \mathrm{C}$ for $1 \mathrm{~min}$, at $40^{\circ} \mathrm{C}$ (REP-PCR) for $1 \mathrm{~min}$ and at $65^{\circ} \mathrm{C}$ for $8 \mathrm{~min}$, followed by a final extension of $16 \mathrm{~min}$ at $65^{\circ} \mathrm{C}$. PCR products $(10 \mu 1)$ were separated on $2 \%$ agarose gel (sigma) containing $1 \times$ TBE buffer $(0.1$ mol.L-1 Tris-HCL, 0.1 mol.L- 1 boric acid, 0.002mol.L-1 EDTA, PH= 8) at 4 V.CM-1. After rep-PCR, gel images were transferred to Phoretix 1D software. Rolling ball method was used to remove the background. Identification of the bands was performed manually. The standard strain (E. coli ATCC 25922) was used to determine the lines Rf (standardization bands between different samples). Bands matching with error rate of 0.02 were done. Code 0 (no band) and 1(band) from the program were transferred to NTSYSpc software and finally drew a dendrogram of Dice similarity coefficient with the Complete Linkage algorithm.

\section{Statistical data analysis}

Data was analyzed by employment of Graph Pad Prism version 6.1, which t-test and F-test were applied where needed. The confidence interval equal to $95 \%(\mathrm{CI}=95 \%)$ was considered and $\mathrm{P}$ value $<0.05$ was significant.

\section{Results}

\section{Susceptibility pattern}

Percentage of isolates resistant to antibiotics in this survey in the disk diffusion method was as following: FOX 46.5\%, CAZ 45\%, CTX $72 \%$, CPM $4.5 \%$, ATM $39.5 \%$, ERY $93.5 \%$, GM $36.5 \%$, TE $75 \%$, SXT 57\%, AX 85\%, AM 91\%, AN 15.5\%, IPM 0.5\%, and CP 39\%. The majority $(\mathrm{n}=76)$ were resistant to Ampicillin, whereas the lowest resistance was shown to imipenem.

\section{CAZ and CTX MIC of isolates}

Serial concentrations of 0.5 to $\geq 512 \mu \mathrm{g} / \mathrm{ml}$ of Ceftazidime were prepared and tested. Strains resistant to Ceftazidime MIC for the combination were Clavulanic acid. In this experiment, 90 isolates of $\mathrm{K}$. pneumonia and MICCAZ $\geq 16$ were considered resistant. The use of Clavulanic acid inhibitors this number was reduced to 18 isolated (Table 1) (Table 2). Cefotaxime MIC levels among the isolates have been shown in Table 2. Cefotaxime MIC for the resistant strains was done with and without Clavulanic acid. In this test, 145 isolates with MIC CTX $\geq 4$ were detected and 65 isolates were Clavulanic acid susceptible.

Table I Ceftazidime MIC in the presence and without the presence of Clavulanic acid for K. pneumonia isolates.

\begin{tabular}{lllllll}
\hline $\boldsymbol{\mu g} / \mathbf{m l}$ & $\leq \mathbf{4}$ & $\mathbf{8}$ & $\mathbf{1 6 - 3 2}$ & $\mathbf{6 4 - 1 2 8}$ & $\mathbf{2 5 6 - 5 1 2}$ & $>\mathbf{5 1 2}$ \\
\hline $\mathrm{CAZ}$ & 83 & 27 & 66 & 13 & $\mathrm{I}$ & 10 \\
$\mathrm{CAZ}+\mathrm{CA}$ & 149 & 33 & 8 & 4 & 2 & 4
\end{tabular}

Table 2 Cefotaxime MIC in the presence and without the presence of Clavulanic acid for E. coli isolates.

\begin{tabular}{llllllll}
\hline $\boldsymbol{\mu g} / \mathrm{ml}$ & $1 \geq$ & $\mathbf{2}$ & $\mathbf{8 - A p r}$ & $\mathbf{1 6 - 3 2}$ & $\mathbf{6 4 - 1 2 8}$ & $\mathbf{2 5 6 - 5 1 2}$ & $\geq \mathbf{5 1 2}$ \\
\hline CTX & 49 & 6 & 48 & 21 & 34 & 21 & 21 \\
CTX+CA & 129 & 6 & 58 & 0 & 1 & 0 & 6 \\
\hline
\end{tabular}

\section{PCR test for the blaTEM gene}

Eighty-four (54.1\%) of 155 isolates were examined for TEM enzyme gene. The highest percentage of this gene was detected in urine samples and the lowest percentage was in blood. The distribution of TEM type enzyme in various clinical origins has been exhibited in Figure 1. Percentage Of antibiotic resistance in isolates with TEM positive has been shown in Figure 2. PCR products for TEM-lactamase gene has been shown in Figure 3. Rep-PCR typing of isolates containing TEM gene. Dendrogram plotted using the Complete Linkage algorithm and Dice similarity coefficient Phoretix 1D software and NTSYSpc (version 2.02) for the TEM gene showed that K. pneumonia placed in 6 clusters (A-F, Figure 4). Most of urinary isolates placed in cluster A showing a genetic relation among them. Moreover, all were susceptible to imipenem and the majority to amikacin. These isolates showed MICCAZ and MICCTX equal or lower than $16 \mu \mathrm{g} / \mathrm{ml}$. The homology of more than $90 \%$ was observed among the majority of isolates and two of them showed 100\% homology and were isolated from wound infection. The phenotypic similarity of these two isolates was also more than $90 \%$. Isolates within each of other clusters also showed similarities like these descriptions (Figure 5). A similarity of $90 \%$ was observed among 50 $80 \%$ of isolates. The relation of rep-PCR clusters to drug resistance and infection sites has been depicted in Table 3 . 
Table 3 The relation of rep-PCR clusters to drug resistance and infection sites.

\begin{tabular}{|c|c|c|c|c|c|c|}
\hline Variable & $\begin{array}{l}\text { Cluster A } \\
(n=21)\end{array}$ & $\begin{array}{l}\text { Cluster B } \\
(n=8)\end{array}$ & $\begin{array}{l}\text { Cluster C } \\
(n=7)\end{array}$ & $\begin{array}{l}\text { Cluster D } \\
(n=19)\end{array}$ & $\begin{array}{l}\text { Cluster E } \\
(n=6)\end{array}$ & $\begin{array}{l}\text { Cluster } F \\
(n=19)\end{array}$ \\
\hline MDR strains $(n=30)$ & 9 & 3 & I & 9 & I & 8 \\
\hline Stool $(n=11)$ & 3 & I & 0 & 5 & 0 & 2 \\
\hline Urine $(n=24)$ & 9 & I & I & 4 & 2 & 7 \\
\hline Blood $(n=6)$ & 4 & I & 0 & I & 0 & 0 \\
\hline Tissue $(n=13)$ & 4 & 0 & I & 2 & 2 & 4 \\
\hline Wound $(n=17)$ & 1 & 2 & 3 & 4 & 2 & 5 \\
\hline Secretions $(n=9)$ & 0 & 3 & 2 & 3 & 0 & 1 \\
\hline
\end{tabular}

MDR, multiple drug resistant; ICU, intensive care unit; ID, infectious disease

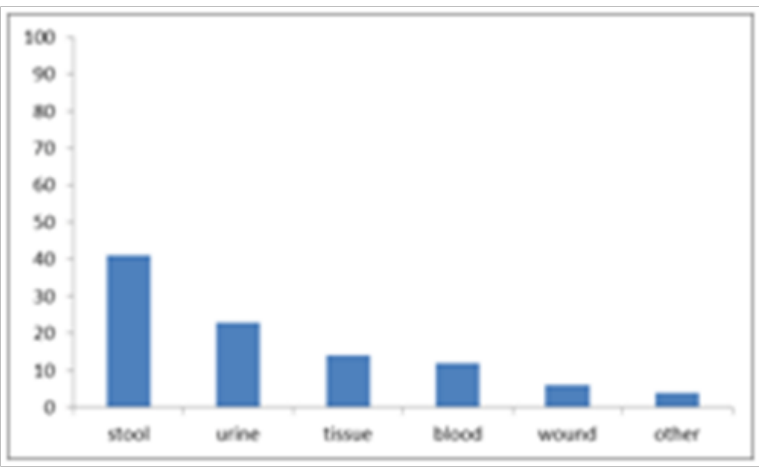

Figure I Percentage distribution of TEM beta-lactamase enzymes in different clinical samples.

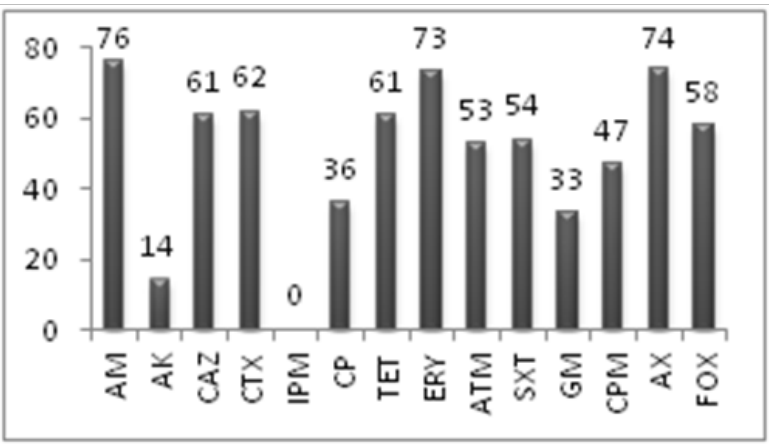

Figure 2 Percentage Of antibiotic resistance in TEM positive isolates.

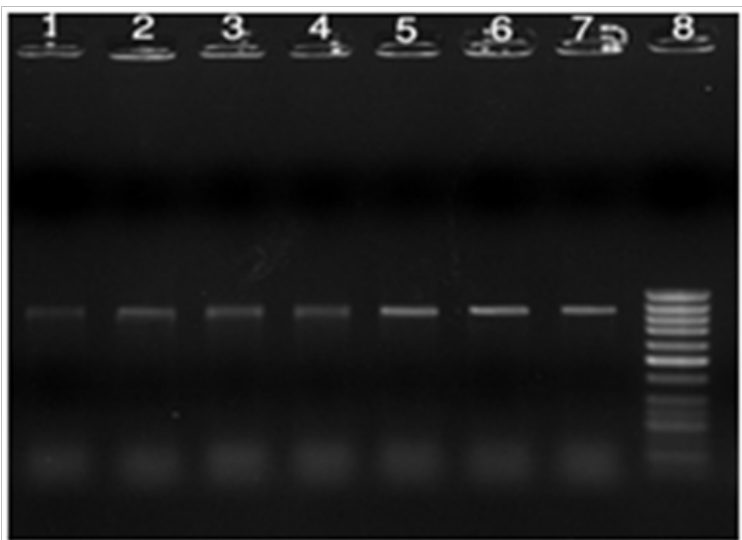

Figure 3 PCR products of TEM gene with 850bp size, Lanes 1-6: positive clinical samples, Lane 7: positive control, and lane 8: 100bp DNA Ladder.

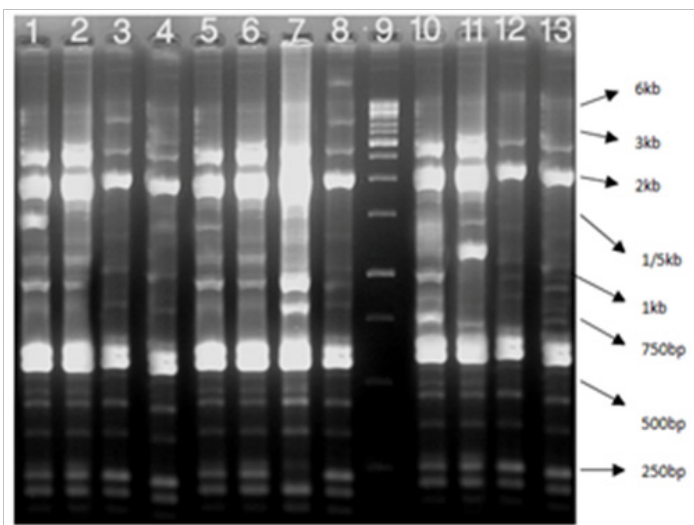

Figure 4 Rep-PCR reactions for genotyping of TEM positive K. pneumonia: Lane I positive control E. coli ATCC25922, Lane 2-I I clinical samples, and Lane 12 I kb DNA Ladder.

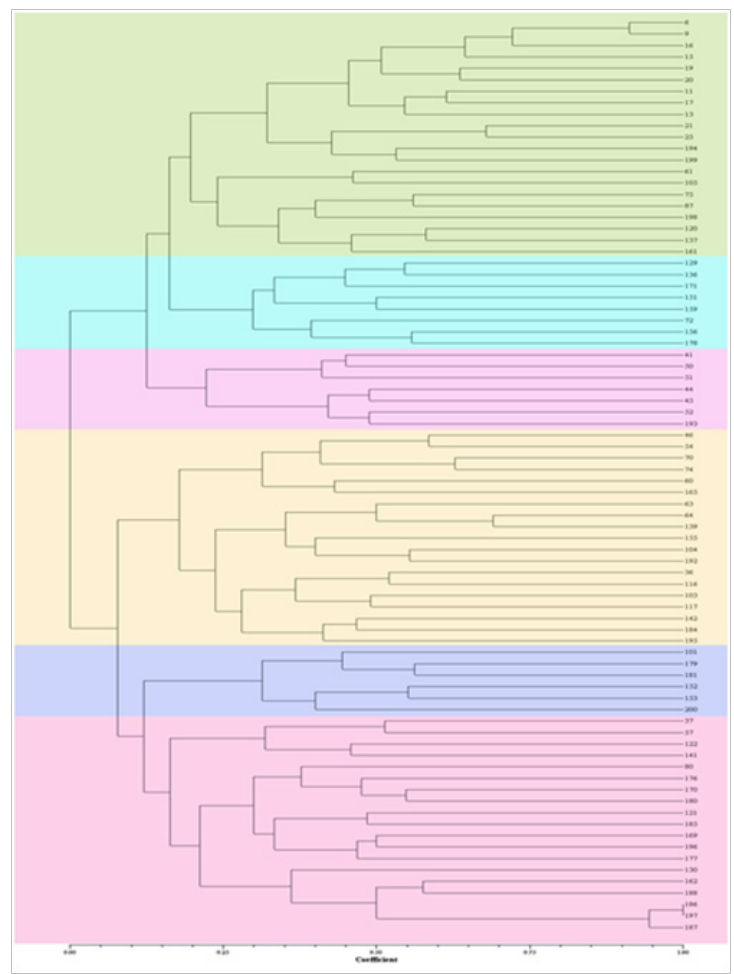

Figure 5 The dendrogram of six clusters (A-F) obtained for TEM-positive isolates. 


\section{Discussion}

In this study, among hospitalized patients the majority of isolates were resistant to ampicillin, erythromycin, co-amoxiclav, but more than $90 \%$ were susceptible to imipenem. Other studies have also shown a high resistance to these antimicrobials worldwide. ${ }^{24,25}$ However carbapenem-resistant $\mathrm{K}$. pneumonia has been frequently isolated and developed. ${ }^{26,27}$ Resistance to amikacin was observed among $15.5 \%$ of isolates. We observed a correlation between antibiotic susceptibility and each rep cluster. A study by Duin ${ }^{28}$ showed a relation between rep cluster type A and KPC producing $\mathrm{K}$. pneumonia, in which most of positive isolates placed in cluster A, although two isolates showed different susceptibilities to amino glycosides and tige cyclones. In this study, 76/200 (87.3\%) of isolates amplified the blaTEM gene. The rep-PCR pattern depicted six clusters (A-F), which mostly classified in clusters $\mathrm{A}, \mathrm{D}$ and $\mathrm{F}$, whereas aminority classified in clusters $\mathrm{B}$ and $\mathrm{C}$. The genetic background of isolates was different in spite of $90 \%$ similarity of $50-80 \%$ of them and thus it was suggested a polyclonal spread of TEM type producing isolates in a study from Portugal, CTX-M and TEM type producing K. pneumonia isolates were genetically diverse. ${ }^{29}$ In Mensah' study in Ghana, $48.1 \%$ of $\mathrm{K}$. pneumonia and $E$. coli isolates were TEM positive, but the genetic relationship of isolates has not been investigated. ${ }^{30}$ To the best of our knowledge no previous studies have determined genetic relationship among TEM type producing $\mathrm{K}$. pneumonia isolates. In a study by Shakibaei from Kerman, only $2.5 \%$ of $\mathrm{K}$. pneumonia isolates amplified TEM enzyme, but strain typing has not been elucidated. ${ }^{31}$ Another study by Mansury ${ }^{32}$ from Shiraz exhibited that $16 \%$ of $\mathrm{K}$. pneumonia amplified TEM, but no typing method was performed. In a study in Tehran, among 43K. Pneumonia (14 ESBL positive and 29 ESBL negative) which adopted for RAPD-PCR typing, 46.5\% belonged to a single profile (genotype 1), of which, the majority $(62.1 \%)$ were non-ESBL producers. ${ }^{33}$

We observed that TEM type isolates from urine and blood specimens were mostly classified in cluster A (13/30), and those from stool, wound and secretions were classified in cluster D. moreover, 16 isolates from urine $(n=7)$, tissue $(n=4)$ and wound $(n=5)$ were classified in cluster $\mathrm{F}(16 / 54)$. A study by Kathrin have exhibited that there is a possibility of relation between site of infections and genetic background, drug resistance or pathogenesis of Klebsiella spp..$^{34}$ We appreciated that MDR K. pneumonia (isolates with resistance to beta lactams, quinolones and amino glycosides, $n=30$ ) distributed in 6 clusters in a diverse pattern. The limitations of this study were lack of typing with more accurate or reliable methods such as pulsed field gel electrophoresis (PFGE) and multi-locus sequence typing (MLST), no data of hospital settings for some reasons, low number of TEM type producing $\mathrm{K}$. pneumonia isolates and a narrow area of study.

\section{Conclusion}

The findings of the current study concluded the emergence and spread of K. pneumonia isolates producing TEM type enzymes with a genetically diverse background in Tehran.

\section{Ethical approval}

This study was approved by ethical committee of Bandar Abbas University of Medical Sciences, (Grant No. 1649547/6743, 2015).

\section{Acknowledgements}

None.

\section{Conflict of interest}

The author declares no conflict of interest.

\section{References}

1. Talbot GH, Bradley J, Edwards JE, et al. Bad bugs need drugs:an update on the development pipeline from the Antimicrobial Availability Task Force of the Infectious Diseases Society of America. Clinical Infectious Diseases. 2006;42(5):657-668.

2. Bouchillon SK, Badal RE, Hoban DJ, et al. Antimicrobial susceptibility of inpatient urinary tract isolates of Gram-negative bacilli in the United States: Results from the Study for Monitoring Antimicrobial Resistance Trends (SMART) Program:2009-2011. Clinical therapeutics. 2013;35(6):872-877.

3. Peirano G, Sang JHK, Pitondo-Silva A, et al. Molecular epidemiology of extended-spectrum- $\beta$-lactamase-producing Klebsiella pneumoniae over a 10 year period in Calgary, Canada. J Antimicrob Chemothe. 2012;67(5):1114-1120

4. Wang G, Huang T, Surendraiah PKM, et al. CTX-M $\beta$-Lactamaseproducing Klebsiella pneumoniae in Suburban New York, New York, USA. Emerg Infect Dis. 2013;19(11):1803-1810.

5. Novais Â, Baquero F, Machado E, et al. International spread and persistence of TEM-24 is caused by the confluence of highly penetrating Enterobacteriaceae clones and an IncA/C2 plasmid containing Tn1696::Tn1 and IS5075-Tn21. Antimicrob Agents Chemother. 2010;54(2):825-834

6. Partridge SR, Hall RM. Evolution of transposons containing blaTEM genes. Antimicrobial agents and chemotherapy. 2005;49(3):1267-1268.

7. Kiratisin P, Apisarnthanarak A, Laesripa C, et al. Molecular characterization and epidemiology of extended-spectrum- $\beta$-lactamaseproducing Escherichia coli and Klebsiella pneumoniae isolates causing health care-associated infection in Thailand, where the CTX-M family is endemic. Antimicrob Agents Chemother. 2008;52(8):2818-2824.

8. Paterson DL, Bonomo RA. Extended-spectrum $\beta$-lactamases: a clinical update. Clin Microbiol Rev. 2005;18(4):657-686.

9. Tobes R, Ramos JL. REP code: defining bacterial identity in estrogenic space. Environ Microbiol. 2005;7(2):225-228.

10. Shahcheraghi F, Moezi H, Feizabadi MM. Distribution of TEM and SHV beta-lactamase genes among Klebsiella pneumoniae strains isolated from patients in Tehran. Med Sci Monit. 2007;13(11):BR247-BR50.

11. Eftekhar F, Rastegar M, Golalipoor M, et al. Detection of Extended Spectrum B-Lactamases in Urinary Isolates of Klebsiella pneumoniae in Relation to Bla ,Bla and Bla Gene Carriage. Iran J Public Health. 2012;41(3):127-132.

12. Feizabadi MM, Delfani S, Raji N, et al. Distribution of bla TEM, bla SHV, bla CTX-M genes among clinical isolates of Klebsiella pneumoniae at Labbafinejad Hospital, Tehran, Iran. Microb Drug Resist. 2010;16(1):49-53

13. Nasehi L, Shahcheraghi F, Nikbin VS, et al. PER, CTX-M, TEM and SHV Beta-lactamases in clinical isolates of Klebsiella pneumoniae isolated from Tehran, Iran. Iranian Journal of Basic Medical Sciences. 2010;13(3):111-118

14. Feizabadi MM, Mahamadi-Yeganeh S, Mirsalehian A, et al. Genetic characterization of ESBL producing strains of Klebsiella pneumoniae from Tehran hospitals. J Infect Dev Ctries. 2010;4(10):609-615.

15. Ghasemi Y, Archin T, Kargar M, et al. A simple multiplex PCR for assessing prevalence of extended-spectrum $\beta$-lactamases producing Klebsiella pneumoniae in Intensive Care Units of a referral hospital in Shiraz, Iran. Asian Pac J Trop Med. 2013;6(9):703-708. 
16. Ishii S, Sadowsky MJ. Applications of the rep-PCR DNA fingerprinting technique to study microbial diversity, ecology and evolution. Environ Microbiol. 2009;11(4):733-740.

17. Hernández J, Martinez-Martinez L, Cantón R, et al. Nationwide study of Escherichia coli and Klebsiella pneumoniae producing extendedspectrum $\beta$-lactamases in Spain. Antimicrob Agents Chemother. $2005 ; 49(5): 2122-2125$.

18. Zowawi HM, Sartor AL, Balkhy HH, et al. Molecular characterization of carbapenemase-producing Escherichia coli and Klebsiella pneumoniae in the countries of the Gulf cooperation council: dominance of OXA-48 and NDM producers. Antimicrob Agents Chemother. 2014;58(6):30853090 .

19. Naas T, Cuzon G, Robinson A, et al. Neonatal infections with multidrugresistant ESBL-producing E. cloacae and K. pneumoniae in Neonatal Units of two different Hospitals in Antananarivo, Madagascar. BMC Infect Dis. 2016;16(1):1.

20. Giske CG, Fröding I, Hasan CM, et al. Diverse sequence types of Klebsiella pneumoniae contribute to the dissemination of blaNDM-1 in India, Sweden, and the United Kingdom. Antimicrob Agents Chemother. 2012;56(5):2735-2738.

21. Hombach M, Mouttet B, Bloemberg GV. Consequences of revised CLSI and EUCAST guidelines for antibiotic susceptibility patterns of ESBLand AmpC $\beta$-lactamase-producing clinical Enterobacteriaceae isolates. $J$ Antimicrob Chemother. 2013;68(9):2092-2098.

22. Livermore DM, Brown DF. Detection of $\beta$-lactamase-mediated resistance. Journal of Antimicrobial Chemotherapy. J Antimicrob Chemother. 2001;48(suppl 1):59-64.

23. Versalovic J, Koeuth T, Lupski R. Distribution of repetitive DNA sequences in eubacteria and application to finerpriting of bacterial enomes. Nucleic Acids Res. 1991;19(24):6823-6831.

24. Maina D, Makau P, Nyerere A, et al. Antimicrobial resistance patterns in extended-spectrum $\beta$-lactamase producing Escherichia coli and Klebsiella pneumoniae isolates in a private tertiary hospital, Kenya. Microbiology Discovery. 2013;1(1):5.

25. Sanchez GV, Master RN, Clark RB, et al. Klebsiella pneumoniae antimicrobial drug resistance, United States, 1998-2010. Emerg Infect Dis. 2013;19(1):133-136.
26. Kontopidou F, Giamarellou H, Katerelos P, et al. Infections caused by carbapenem-resistant Klebsiella pneumoniae among patients in intensive care units in Greece: a multi-centre study on clinical outcome and therapeutic options. Clin Microbiol Infect. 2014;20(2):O117-O123.

27. Riyahi Zaniani F, Meshkat Z, Naderi Nasab M, et al. The prevalence of TEM and SHV genes among extended-spectrum beta-lactamases producing Escherichia coli and Klebsiella pneumoniae. Iran J Basic Med Sci. 2012;15(1):654-660.

28. Van Duin D, Perez F, Rudin SD, et al. Surveillance of carbapenemresistant Klebsiella pneumoniae: tracking molecular epidemiology and outcomes through a regional network. Antimicrob Agents Chemother. 2014;58(7):4035-4041.

29. Rodrigues C, Machado E, Ramos H, et al. Expansion of ESBL-producing Klebsiella pneumoniae in hospitalized patients: A successful story of international clones (ST15, ST147, ST336) and epidemic plasmids (IncR, IncFII K). Int J Med Microbiol. 2014;304(8):1100-1108.

30. Oduro-Mensah D, Obeng-Nkrumah N, Bonney EY, et al. Genetic characterization of TEM-type ESBL-associated antibacterial resistance in Enterobacteriaceae in a tertiary hospital in Ghana. Ann Clin Microbiol Antimicrob. 2016;15(1):1.

31. Shakibaie MR, Shahcheraghi F, Hashemi A, et al. Detection of TEM, SHV and PER Type Extended-Spectrum B-Lactamase Genes among Clinical Strains of Pseudomonas aeruginosa Isolated from Burnt Patients at Shafa-Hospital, Kerman, Iran. Pak J Med Sci. 2008;11(2):104-111.

32. Mansury D, Motamedifar M, Sarvari J, et al. Antibiotic susceptibility pattern and identification of extended spectrum $\beta$-lactamases (ESBLs) in clinical isolates of Klebsiella pneumoniae from Shiraz, Iran. Iran J Microbiol. 2016;8(1):55-61.

33. Eftekhar F, Nouri P. Correlation of RAPD-PCR Profiles with ESBL Production in Clinical Isolates of Klebsiella pneumoniae in Tehran. $J$ Clin Diagn Res. 2015;9(1):DC01-DC03.

34. Herzog KA, Schneditz G, Leitner E, et al. Genotypes of Klebsiella oxytoca isolates in nosocomial pneumonia is distinct from antibiotic-associated hemorrhagic colitis isolates. J Clin Microbiol. 2014;52(5):1607-1616. 